\title{
Congenital divided naevus of the eyelids
}

\author{
P J MCDONNELL' AND B J MAYOU \\ From the 'Department of Ophthalmology and the ${ }^{2}$ Department of Plastic Surgery, St Thomas's Hospital, \\ London
}

SUMMARY A newborn infant presenting with a divided congenital melanocytic naevus of the eyelid is described. Because of the severe disfigurement, risk of later malignant change in the lesion, and the possibility of deprivation amblyopia, early surgical treatment is recommended for all medium and large congenital melanocytic naevi of the eyelid. Surgery in the first few months of life gives the best cosmetic results.

Divided naevus of the eyelids is a rare form of congenital melanocytic naevus that involves the upper and lower eyelids of one eye. The naevus is present in contiguous areas of the upper and lower lid margins, so that when the eyelids are closed the eye appears to be covered by one large naevus.

Congenital divided naevus of the eyelids was first described in 1919 by Fuchs.' Since then about 30 cases have been reported. All were children or adults at the time of presentation. We believe this is the first case report of a baby with divided naevus presenting at birth to the ophthalmologist and plastic surgeon, and receiving early surgical treatment.

Correspondence to Mr P J McDonnell, FRCS, South Wing Eye Department, St Thomas's Hospital, Lambeth Palace Road, London SE1 7EH.

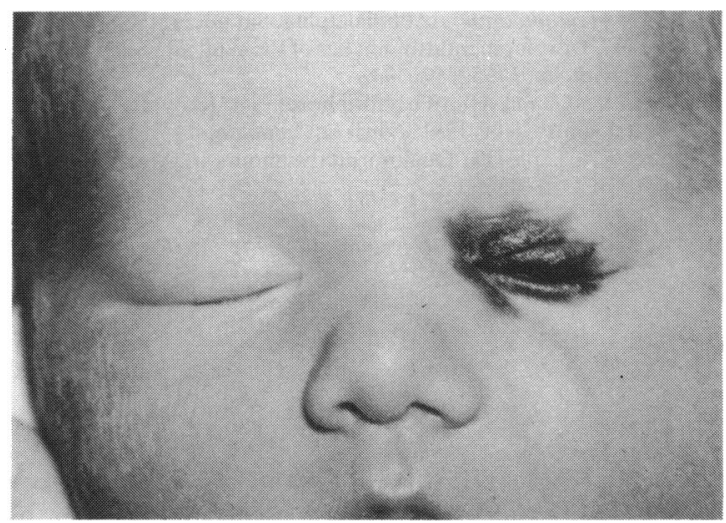

Fig. 1 Clinical photograph showing the thickened and darkly pigmented naevus involving the upper and lower lids and medial canthus of the left eye.

\section{Case report}

The patient was a female infant born on 27 June 1986 by normal delivery. She weighed $3.2 \mathrm{~kg}$, and appeared healthy, with no abnormalities visible apart from a pigmented skin lesion round the left eye (Fig. 1). There were no other pigmented skin lesions and no relevant family history. The lesion had a vertical diameter of $2 \mathrm{~cm}$ and a horizontal diameter of $2.5 \mathrm{~cm}$. The skin was darkly pigmented and thickened and involved the medial three-quarters of the upper and lower lid and the medial canthus. There was also some pigmentation of the tarsal conjunctiva of both lids. The bulbar conjunctiva and the sclera were normal. There was a slight left ptosis due to the thickening of the upper lid, but the pupil was not covered. The rest of the eye was normal; in particular the fundus showed no abnormal choroidal pigmentation. A diagnosis of congenital divided melanocytic naevus of the eyelids was made.

On 10 July 1986 dermabrasion of the naevus was carried out under general anaesthetic, and a punch biopsy of the skin was taken. Histological examination showed a congenital melanocytic naevus with prominent junctional activity and marked pigmentation (Fig. 2). The naevus was markedly debulked by the dermabrasion and at first the pigmentation was much less (Fig. 3). However, over the next four weeks the pigmentation returned and the naevus bulk increased to its former size (Fig. 4). On 20 August 1986 the naevus was excised and the skin replaced with full-thickness skin grafts taken from behind the ear. The conjunctival pigmentation was not excised.

Postoperative assessment at two months showed 


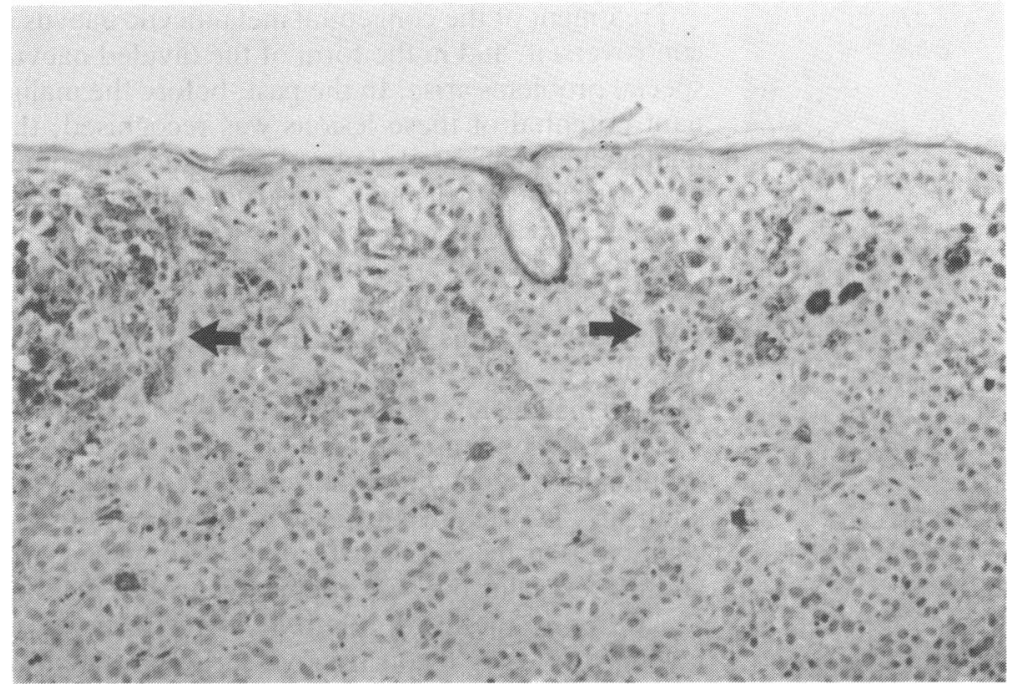

Fig. 2 Photomicrograph of punch biopsy specimen showing nests of naevus cells (arrows) in the dermis. Haematoxylin and eosin.

good lid closure and a satisfactory graft, with no recurrence of pigmentation (Fig. 5). The visual axis was clear and no deviation was demonstrated on cover test.

\section{Discussion}

Congenital melanocytic naevi occur in about $1 \%$ of the population. They may be divided into three groups ${ }^{2}$ according to size: (1) small (less than $1.5 \mathrm{~cm}$ in diameter), (2) medium $(1.5 \mathrm{~cm}$ to $20 \mathrm{~cm}$ in diameter), and (3) large (greater than $20 \mathrm{~cm}$ in diameter). The medium and large naevi have similar clinical features, which include a thickened irregular surface, increased pigmentation giving a dark brown colour, and varying amounts of abnormal hair growth. The small naevi are usually flat and only lightly pigmented. Congenital melanocytic naevi often become larger, thicker, and more hairy in

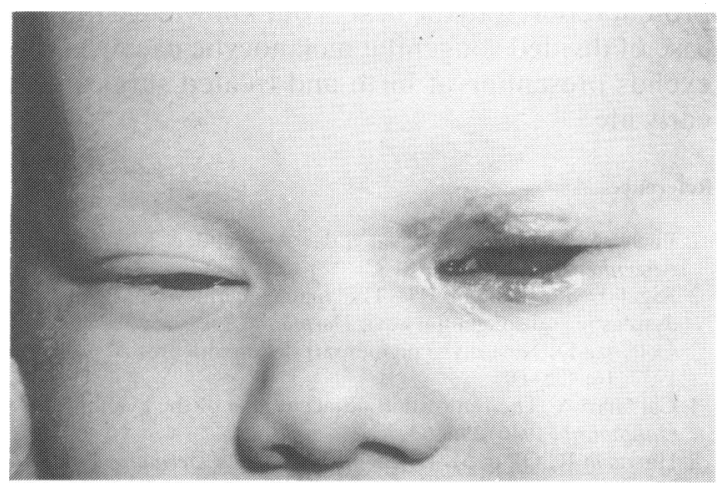

Fig. 3 Appearance of the left eye one week after dermabrasion with minimal pigmentation visible. childhood and early adult life, and the lesion may be very disfiguring. Divided naevi of the eyelids are usually medium sized and involve contiguous portions of the upper and lower lid. They are very rare, and only about 30 cases have been reported.

In the first series to be reported Fuchs' described six patients, the youngest of whom was 4 years, with divided naevi. No patients received active treatment. In 1937 Collenza $^{3}$ reported on two patients with divided naevi: a 19-year-old girl and a 26-year-old man, both of whom were treated by excision of the naevus and repair with full-thickness postauricular skin grafts. Callahan, ${ }^{4}$ and Harrison and Okun ${ }^{5}$ reported single cases. The most recent series was

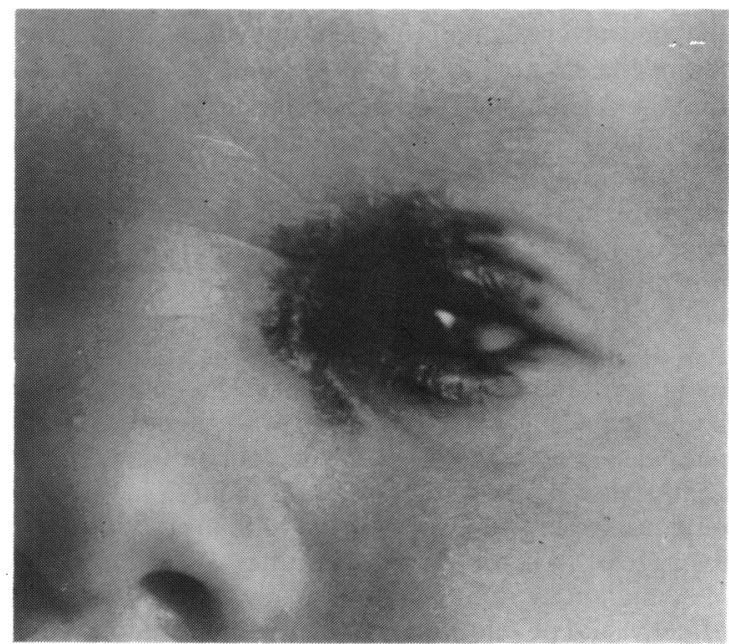

Fig. 4 Five weeks after dermabrasion there is a marked return of the pigmentation. 


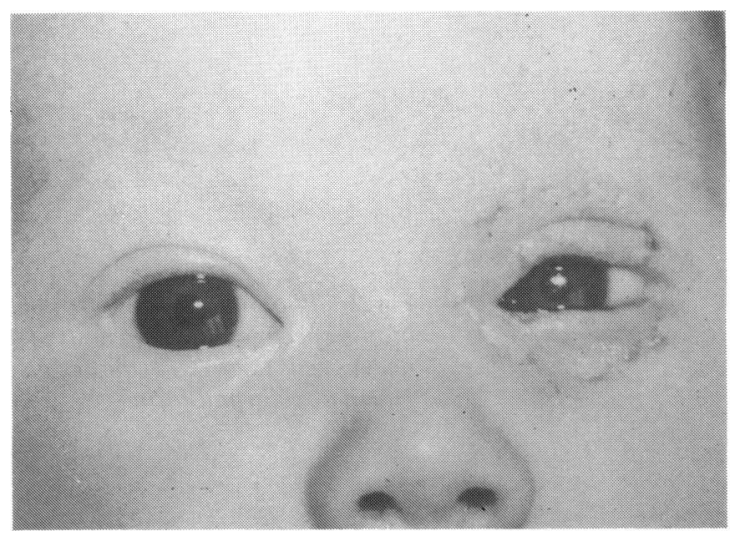

Fig. 5 Appearance of the left eye following excision of the naevus and full thickness skin grafts to the lids.

reported by Ehlers ${ }^{6}$ in 1969. He described 10 cases, the youngest patient being a 15-year-old boy: two patients had simple excision, two needed fullthickness skin grafts to repair the defect, two were treated with cryotherapy, and the rest received no treatment. There are no cases in the literature of a patient presenting at birth to the ophthalmologist.

The divided naevus is thought to arise during fetal development at a time when the eyelids are fused. The eyelids first appear as ectodermal protrusions at 6 weeks of fetal development (12 $\mathrm{mm}$ stage). They grow towards each other and gradually fuse, with the process being complete at 9 weeks ( $40 \mathrm{~mm}$ stage). Lipid then starts to appear in the junctional zone at about 15 weeks, but the eyelids stay fused until about 24 weeks (the $150 \mathrm{~mm}$ stage), when they gradually separate. $^{7}$

Studies have shown that melanocytes migrate from the neural crest and reach human fetal epidermis about the 12- to 14 -week stage,$^{8}$ and it seems likely that the divided melanocytic naevus arises just before the 15th week of fetal development.

Congenital melanocytic naevi can cause a number of problems for the patient. The appearance of the naevus is very disfiguring and, if in a prominent position, often causes problems of cosmesis, particularly in childhood and adolescence. There is a definite risk of malignant change in the naevus giving rise to malignant melanoma, which is best documented for large naevi but is thought to exist for medium sized naevi as well. ${ }^{9}$ The reported incidence of malignant change is very variable, ranging from $2 \%$ to $30 \%$ depending on the length of follow-up, with an average of $14 \%$ for a whole lifetime.

A melanocytic naevus involving the eyelids may affect visual development if the increased bulk of the upper lid causes a mechanical ptosis and occlusion of the visual axis. ${ }^{10}$
Treatment of the congenital melanocytic naevus is controversial, and in the form of the divided naevus special problems arise. In the past, before the malignant potential of these lesions was recognised, the management consisted of observation and sometimes late surgical removal and reconstruction for cosmetic reasons. However, with the realisation of the risk of malignant change and the observation that most cases of prepubertal melanoma arising in congenital melanocytic naevus occur in the first 3 to 5 years of life, the emphasis has changed to a more aggressive surgical management of these patients. ${ }^{11}$

The cosmetic appearance of surgical scars is usually poor in childhood and improves throughout adult life. However, the first few months of life are characterised by excellent healing of surgical scars, and this explains the surgical preference for cleft lip repair before the third month of life. We therefore favour very early reconstructive surgery to achieve the best cosmetic result. Dermabrasion has been reported to be a successful technique for removing congenital melanocytic naevus if the lesion is treated early in life and the naevus cells are still limited to the superficial dermis. ${ }^{12}{ }^{13}$ This technique uses a metal burr attachment to a dental drill which rotates at high speed and is used to abrade the epidermis and superficial dermis. Unfortunately in our patient the punch biopsy done at the time of the dermabrasion revealed that the naevus cells were already deep in in the dermis, and it was not surprising that the lesion recurred fairly quickly. If the naevus involves the deep dermis and subcutaneous tissue, then the treatment consists of full-thickness excision followed by repair with full-thickness skin graft or split skin graft depending on the size of the defect.

Large naevi involving the eyelids may have to be treated in stages. ${ }^{14}$ Divided naevi which are excised are most conveniently repaired with a full-thickness postauricular skin graft. Five cases of divided naevi treated by this procedure have been reported. ${ }^{36}$ The present report is to the best of our knowledge the first case of divided congenital melanocytic naevus of the eyelids presenting at birth and treated surgically in early life.

\section{References}

1 Fuchs A. Ueber geteilte Naevi der Augenlider. Klin Monatsbl Augenheilkd 1919; 63: 678-83.

2 Rigel DS, Friedman RJ. The management of patients with dysplastic and congenital nevi. Dermatol Clin 1985; 3: 251-5.

3 Collenza D. Nevi divisi pigmentari delle palpebre. Boll Oculist 1937; 16: 435-60.

4 Callahan A. The removal of adjacent nevi of the eyelids. Am J Ophthalmol 1946; 29: 563-5.

5 Harrison R, Okun M. Divided nevus. Arch Dermatol 1960; 82: 235-6.

6 Ehlers N. Divided nevus. Acta Ophthalmol (Kbh) 1969; 47: 1004-11. 
7 Andersen H, Ehlers N, Matthiessen ME. Histochemistry and development of the human eyelids. Acta Ophthalmol (Kbh) 1965; 43: 642-68.

8 Breathnach AS, Wyllie LM. Electron microscopy of melanocytes and Langerhans cells in human fetal epidermis at fourteen weeks. J Invest Dermatol 1965; 44: 51-60.

9 Kaplan EN. The risk of malignancy in large congenital nevi. Plast Reconstr Surg 1974; 53: 421-8.

10 Antinone RL, Helveston EM, Bennett JE, Keener P. Giant hairy nevus: preventable cause of amblyopia. $J$ Pediatr Ophthalmol 1976; 13: 192-5.

11 Arons MS, Hurwitz S. Congenital nevocellular nevus: a review of the treatment controversy and a report of 46 cases. Plast Reconstr Surg 1983; 72: 355-65.

12 Johnson HA. Permanent removal of pigmentation from giant hairy naevi by dermabrasion in early life. Br J Plast Surg 1977; 30: 321-3.

13 Miller CJ, Becker DW. Removing pigmentation by dermabrading naevi in infancy. Br J Plast Surg 1979; 32: 124-6.

14 de Dulanto F, Camacho-Martinez F. Sanchez-Muros J, de Cosme L. A giant hairy pigmented nevus on the face: excision and reconstruction in stages. J Dermatol Surg Oncol 1979; 5: 215-8.

Accepted for publication 7 January 1987. 